\title{
Problems and Prospects for Quantum Computational Speed-up
}

\author{
E.V. Krishnamurthy \\ Computer Sciences Laboratory, \\ Australian National University, Canberra, ACT 0200, Australia. \\ abk@discus.anu.edu.au
}

\begin{abstract}
This paper studies the problems involved in the speed-up of the classical computational algorithms using the quantum computational paradigm. In particular, we relate the primitive recursive function approach used in computability theory with the harmonic oscillator basis used in quantum physics. Also, we raise some basic issues concerning quantum computational paradigm: these include failures in programmability and scalability, limitation on the size of the decoherence - free space available and lack of methods for proving quantum programs correct. In computer science, time is discrete and has a well-founded structure. But in physics, time is a real number, continuous and is infinitely divisible; also time can have a fractal dimension. As a result, the time complexity measures for conventional and quantum computation are incomparable. Proving properties of programs and termination rest heavily on the well-founded properties, and the transfinite induction principle. Hence transfinite induction is not applicable to reason about quantum programs.
\end{abstract}

\section{Introduction}

Quantum theory of computation is an important area of fundamental study linking the physical quantum theory with the logical theory of computability. From a pragmatic point of view, we are not even sure whether this study will lead us to the design of a quantum computer in the near future or almost never! Although, quantum version of boolean logic circuits $[4,6,8,9,23]$ have been shown to be feasible, the machine hierarchy (namely, finite state machines, push-down stack machine and Turing machine) and the corresponding Chomskian grammatical and linguistic hierarchy [11,13], their connection to algorithms and related data structures have not been established in quantum computational logic, leaving a wide gap in understanding. Yet, some problems have been shown to be amenable for quantum speed-up [4,8,10,23]. These algorithms have been ad hoc discoveries using the principle of quantum superposition (Schrodinger cat states) and are probabilistic. Based on these, there have been several claims that quantum speed-up can break the computational intractability, as well as, the Turing barrier of noncomputability. But so far there has been no success to prove these claims due to the difficulty in bridging a physical theory with the logical computability theory. In fact, extending the recursion theory over the integers to real and complex numbers to include approximate operations that are typically composition of functions and solutions of differential equations [3] do not 
result in newer complexity classes and so the standard computational complexity measures remain intact. Yet another obstacle is set by Physics, due to decoherence. Decoherence prevents coupling with the environment thereby making the quantum speed up ineffective $[2,10,23]$. Thus this is a challenging area for innovative experimental and theoretical research seeking answers to many questions.

The purpose of this paper is to explore the reasons as to why the quantum paradigm is not so easy to extend to all of the classical computational algorithms. Firstly, our arguments based on quantum field theory (second quantization) leads us to the result that quantum speed-up can be effective only for the primitive recursive functions (or the unravelled "for loop" or "count loop" programs), which correspond to the harmonic oscillator functions, and not for total or partial recursive functions ("general while loop" programs). This places a severe restriction on the type of algorithms that can be mapped to gain quantum-speed-up. Secondly, the problem of designing quantum algorithms through superposition is analogous to designing algorithms based on the "unrealistic Concurrent-Write- Exclusive Read PRAM" model [14]. That is we

need a quantum measurement that can extract all of the $2^{\mathrm{m}}$ values of the component functions from their superposition pattern. The only way to extract information from the pattern is through the recognition of joint properties among the values of the function $\mathrm{f}(\mathrm{x})$, for example, the periodicity, using quantum probabilistic means. However, extracting a definite answer from this pattern is highly problem domain dependent and is not easy although, Hadamard or Fourier transform and other filtering or projection techniques are useful for particular problems.

While superposition principle provides space complexity reduction for timeindependent parallel computation, there remains yet another possibility called "Zeno squeezing", in which an infinite amount of computation can be performed in a finite time through a time scale change, since time is a real number in Quantum Physics (See Section 6.5). This aspect remains to be explored in quantum speed-up.

In this paper we consider the following issues:

1.To study why quantum algorithms are hard to design and what are the requirements for realising such algorithms?

2. What are the current open problems and what are the limitations in our knowledge?

The aforesaid studies lead to the following results:

(i) Errors in encoding and difficulty in Mapping: Quantum computation work with a continuum of states and use unitary transformation framework over complex numbers rather than conventional boolean gates. In this sense they are analog;hence inexact and are subject to operational errors. Therefore, realising an arbitrary unitary operator with arbitrary precision is not easy. For example, one of the most common quantum gates rotates a quantum bit by an angle; this can result in error causing operational errors to propagate [24].

Quantum paradigm requires that the problems are converted into a periodic superposition of functions. This approach is different from the mapping of processes to architectures, in the sense, it is a structural transformation of a problem from a given domain to a non-dissipative quantum dynamical system. Also the absence of suitable supporting data structures-such as- stack and other data structural mechanisms required in the design of algorithms are not available.This makes programming conceptually difficult.

(ii) Failure of Programmability: Quantum nonlocality [10, 17, 21] creates nonanticipated interactions resulting in the failure of a total program.So the software 
engineering requirement for modular construction of a whole program from its parts, or the principle of denotational semantics is violated. Thus programming a quantum computer through a recursive set-up is not possible.

(iii) Failure of Composition: Conventional programs are built as a composition of primitive recursive functions over the integers. The quantum programs need to realise such functions using a composition of the different real or complex functions for each subprograms. This would result in both operational errors and dissipation leading to accelerated decoherence. Hence the divide and conquer strategy (or related strategies used in algorithmic engineering) in which we decompose a problem into subproblems and composing the results fails [18].

(iv) Failure of Scalabilitty: Scalability is a metric that tells us how the running time to solve a problem scales or varies with its size. Assume that we use the divide and conquer strategy. If we partition the problem into $\mathrm{N}$ pieces and each such partition is solved by $\mathrm{N}$ different processors simultaneously, and the algorithm is linearly scalable, we expect that the total problem is solvable in the same time as solving a single partition plus some overhead for combining the N solutions. Since each such partition is a different coherent state, their phase coherence is to be preserved before combining the results in order to avoid an accelerated decoherence. The preservation of coherence is difficult if the different partitions are not identical as in nontrivial computations.Hence partitioning a problem and solving is ruled out in quantum methodology.Hence scalability fails and we need bigger and bigger machines to solve larger and larger problems.

(v) Quantum Decoherence in while loop: The reason why we cannot use "while loop" for testing is obvious. Each such test in a loop destroys the coherence. Hence "while loop" programs are forbidden quantum mechanically. However, the unravelled "for loop" computations that are primitive recursive are realisable as coherent harmonic oscillator states. But they cannot be composed due to accelerated decoherence. Some attempts are under way to prevent accelerated decoherence by choosing decoherencefree space so that phase coherence between such states remains intact.

(vi) Proving Quantum Programs Correct: The structure of time, namely, whether it is continuous or discrete, plays an important role in reasoning, verification and proving the correctness of programs. In computer science, time has a discrete well-founded structure, while in physics, it is well accepted that the structure of time is a real number and is continuous and is infinitely divisible. In fact time can have a fractal dimension too. This means the transfinite induction principle is not applicable to reason about quantum programs. As a result, the time complexity for conventional and quantum computation are incomparable.

(vii) Curse of dimensionality: We have shown elsewhere [18] that quantum speed-up cannot obviate the curse of dimensionality encountered in many numerical problems due to quantum entropy arsing from operational and decoherence errors.

(viii) Zeno-squeezing: Is there a physical possibility of Zeno squeezing of time through a time-scale change for quantum speed-up? (Section 6.5)

\section{Quantum Algorithmic Paradigm}

Quantum algorithmic paradigm is the realisation of a physical quantum system whose dynamical evolution takes the system from one of a set of input states to one of a set 
of output states. If we assume that a quantum computer consists of a set of quantum components and a quantum register, the quantum computation can be viewed as a process which consists in preparing the input state $S$ (in) and evolving it to an output state $S$ (out). The output state is a positive map of the input state through a linear operator transformation that results in a superposition of all $\mathrm{m}$ input values into a single state resulting in a pattern formation (Concurrent Write without any constraint unknown to computer science theory). The quantum measurement is then supposed to extract (or Exclusive Read) of all the $2^{\mathrm{m}}$ values from their joint properties, e.g. periodicity. It is assumed that such a process preserves quantum coherence in the register since it is the quantum coherence that provides the required quantum speedup. Thus quantum decoherence should not take place before the reading.

\section{Quantum Evolution, Dissipation, and Coherence}

In $[15,16]$ we studied the relationship between the notion of computability and complexity in computer science, and the notion of integrability in mathematics and physics to understand the complexity aspects of quantum computing. In [15], using the Feynman path integral (FPI) approach we showed that a quantum computer behaves like an analog of an Alternating Turing machine (ATM) exhibiting and-or parallelism by computing the probability amplitude for the reachability (transitive closure) between a given initial and the final algorithmic state. We also stated the two important requirements for the success of quantum evolutionary computation, namely:

1. The evolutionary structure of the exact algorithmic solution of a given mathematical problem is mappable to the "exact unitary" evolutionary structure of a quantum dynamical system leading to the exact spectrum, and these two evolutionary structures have isomorphic histories between the initial, intermediate and final states of the computation. Any approximation would incur degenerative operational errors.

2. The initial, intermediate and final states of the quantum dynamical system are coherent states and the evolution of the hamiltonian preserves coherence. In other words, decoherence does not take place during the progress of computation, and before the final result is read by an observer. This requirement heavily hinges upon the theory of quantum measurements.

The requirements 1 and 2 demand that the evolution is nondissipative,Braun [2] and is also coherence preserving. The requirements, namely, coherence preserving evolution, as well as nondissipative can be achieved if the quantum dynamical system belongs to two important group structures, $S U(2)$ and $S U(1,1),[1,16]$. Thus to have a coherence preserving, unitarily evolving, exact quantum computation it is sufficient that the class of computational problems have models isomorphic to $\mathrm{SU}(2)$ or $\operatorname{SU}(1,1)$ group theoretical structures. This class contains instances of periodic problems mappable to harmonic oscillator, e.g., Shor's factorization, and instances of problems mappable to the permutation groups e.g., sorting, Nielsen and Chuang [23]. Note that for the $\mathrm{SU}(2), \mathrm{SU}(1,1)$ unitary processes (dealing with photon number measurement) in a quantum optical system, Ban [1] shows that the entropy decrease of the physical system is equal to the amount of information extracted from the measurement outcomes. That is there is no loss of information and the photon number is exactly obtained. This class of problems corresponds to synchronous coherently evolving non- 
interacting parallel streams of computations whose hamiltonians are amenable for easy composition and analysis using quantum optical devices and molecular spinresonance devices [23]. However, even for such computations the time scale necessary turns out to be not practical. This is because a million computations are to be carried out and the final results read-off in a thermal time scale time less than $10^{-11}$ second, at a very cold temperature as low as 1 degree Kelvin, Landauer [21]. Thus the unitary quantum dynamical evolution is restricted to solve only problems that can be mapped on to non-dissipative hamiltonian systems that can evolve without decoherence.

\section{Primitive Recursion and Harmonic Oscillator Functions}

Two approaches for modelling computability are $[11,13,14]$ :

(i) Turing machine and (ii) Recursive function.

In the last decade the Turing machine model, which is an operational model has been widely used as the basis for modelling the Quantum mechanical computers, see Deutsch [4], Hey and Allen [9] to prove the universality of quantum computers. We may call the Deutsch approach as the first quantisation approach since it is concerned with the wave aspects of particles (Wavicles) rather than the particle aspect of waves. Although, the Turing machine approach is equivalent to recursive function approach, the latter is more suitable than the former for analysis [17]. The QFT approach is called the second quantisation scheme in Quantum physics.

The quanta of the radiation field corresponds to the non-negative integers and the harmonic oscillator spectra correspond to the recursive computation- with the projection, creation and annihilation operators respectively playing the same role as the Selection, Successor and Predecessor in computability theory. Accordingly this approach [17] relates the classical computational models and the quantum field models more directly than the Turing machine approach used earlier [4,9].

If we identify the successor function used in recursive function theory with the bosonic creation (or construction or raising) operator, and predecessor function with the bosonic annihilation (destruction or lowering) operator, then we can generate all the primitive recursive functions (with the quanta as the basic unit of computation) consisting of the function algebra, consisting of some basic functions and closed under certain operations:

\{ZERO, SUCCESSOR, PREDECESSOR, PROJECTION, COMPOSITION, PRIMITIVE RECURSION, BOUNDED MINIMALIZATION $\}[11,13,17]$.

Thus, the harmonic oscillator plays the same role in the quantum physics, as the primitive recursive function generator does in the theory of computation.

The use of harmonic oscillator as the basis, permits us to only compute primitive recursive functions, but not a total recursive function (such as the Ackerman function) or partial recursive function (a general "while loop") $[11,13,17]$.

The precise counting of bosons or phase determination [17,18] provide mutually exclusive approaches for designing three types of primitive recursive exact digital Quantum algorithms, see [23]. In quantum computers, a string of bits is stored of a system equivalent to $\mathrm{m}$ 2-level systems (quantum bits or q-bits). The general state is then a superposition of states $\mathrm{u}(\mathrm{i})$ where $\mathrm{i}$ runs through 0 to $2^{\mathrm{m}}-1$ values of a classical bit string. If the devices are not error-prone and there is no loss of energy, then such a 
superposition is identifiable with a primitive recursive computable function, if the coefficients in the linear combination are exact numbers or exact phases, since the number-phase relation forbids the exact determination of both [17,18]. We can therefore create a coherent superposition of only phases or amplitudes respectively creating Schrodinger phase cats or amplitude cats, Braun [2]. To avoid operational errors, we need to use exact phases as rational numbers using the prime residue or $\mathrm{p}$ adic rationals $[7,12]$. No speed-up methods are known for fast quantum computer arithmetic.

\subsection{Decoherence-Free Sub-Space (DFS)}

Decoherence is a key ingredient in the transition from quantum to classical mechanics. It has been shown $[2,21,24]$ by that in a macroscopic system the decoherence time scale or the time required for loss of coherence is many orders of magnitude less than the classical time scale over which probabilities evolve. However, some exceptions seem possible and one can look for decoherence free subspaces (DFS). Braun [2] shows that if the dimension of the Hilbert space is $2 \mathrm{~N}$, and superrdiance is present, the dimension of the DFS scales as: $\left[2^{\mathrm{N}+1 / 2} / \sqrt{ } \pi \mathrm{N}\right]$, for large $\mathrm{N}$. That is a fraction $\sqrt{ } 2 / \pi \mathrm{N}$ of the Hilbert space can be decoherence free. This means the input and output domain should remain within the DFS. Obviously, Ackermann function would outgrow any given DFS super-exponentially.

\section{Programmability and Scalability}

In programming theory, we define each language construct in terms of certain mathematical entities (numbers, truth values, functions, operators) that model their meaning. The total meaning of a program is then reconstructed as a composition of the meanings of the individual basic constructs; or "a whole "is described in terms of its "parts". If the program is recursively defined, its meaning is the input-output function corresponding to the least or minimal fixed point of a transformation associated with the program $[13,22]$. This ensures that the program has a well-defined functional relationship between the input and output states and the program halts. This assumption also holds true in classical mechanics where the real states are always local and do not contain statistical correlations, among the states of their local subsystems. However, such an assumption breaks down in quantum systems. The quantum system can exhibit both kinematic and measurement-induced nonlocality. The many particle hamiltonian expression one can work out using recursive function like approach may not obey the basic assumption used in denotational semantics, namely, a composite system is composed of parts and the state of whole is definable in terms of the states of its parts. There are quantum correlation between different subsystems due to the superposition principle. In fact, the whole is more than its parts, even if we assume there is no interaction among the subsystems. Therefore, we cannot partition a quantum system into its parts as is done in computer science and synthesize the system using its parts; this is called non-separability. Hence the structural programmability, and the scalability of the system is lost. 


\section{Time, Proof, and Complexity of Quantum Programs}

Reasoning about programs (verification, proving correctness and termination) is an essential requirement in program design. Such schemes are usually based on the transfinite induction principle that hinges on the theory of nonnegative integers

$[19,20,22]$. Also while reasoning about the complexity of computation, as well as, time dependent and real time programs, time is represented as a discrete set of points mappable to the positive integers or to a set of intervals and temporal logic is used [19]. Such a notion of time satisfies the well-founded relationship to be described in the next subsection. Proving properties of programs and termination rest heavily on the well-founded properties, and chain reasoning method.

Despite numerous attempts to revise this concept over many years, it is well accepted in Physics that time is a real number, continuous and is infinitely divisible. (In fact, time can have a fractal dimension too). Many of the physical theories rest on this fundamental assumption. The notion of time complexity in computation depends on whether the time is continuous or discrete, since this notion distinguishes the continuous time dynamical evolution (as in analog computation) from the discrete digital computation. This is because time appears as an intrinsic parameter in the process of quantum (analog) evolution. For example, under suitable conditions, it may be possible to use an algebraic transformation or rescaling of the time parameter of a differential equation to rearrange it so that that the processes which take infinite time can be executed in a finite time in analog machines. However, analog computing is not effective in the sense of Church-Turing thesis $[3,4,11,13]$. Thus the notion of time complexity is incomparable between the analog and the digital set- up. As an example, consider the mapping: $\mathrm{t}:=(\mathrm{t}-1) / \mathrm{t}$. This would transform the time parameter from the range $[1, \infty]$ to $[0,1]$. This is called Zeno contraction or squeezing (Section 6.5). Such types of contractions, dilatations or scale changes abound in Physics. Thus the computability logic and physics widely differ in defining the structure of time.

\subsection{Well-Founded Sets}

A binary relation $<$ is well-founded over a class of objects, if it satisfies the nodecreasing condition; that is, there is no infinite sequence of objects decreasing with respect to that relation. In other words, there are no sequences $\{x(0), x(1), x(2)$, $x(3), \ldots$.$\} of objects such that: x(0)>x(1)>x(2)>x(3)>\ldots$ where $>$ is the inverse binary relation of $<$. For a detailed study of well founded sets and relations, and related temporal logic, see Manna and Waldinger [22], Kroger [19]. Formally a wellfounded relation (WFR) is defined over a set of objects (obj) thus: There are no infinite sequences $\{t(0), t(1), t(2), \ldots\}$ of objects $t(u)$ such that: for all integers $u$ object $\mathrm{t}(\mathrm{u})$ and for all integers $\mathrm{u}$ : $\mathrm{t}(\mathrm{u})>\mathrm{t}(\mathrm{u}+1)$.

A set in which the elements are related through a well-founded relation is called a well-founded set.

\subsection{Properties of Well-Founded Relation (WFR)}

Irreflexive: That is there are no objects such that $\mathrm{x}<\mathrm{x}$.

Asymmetric: For all object $\mathrm{x}, \mathrm{y}$ : [If $\mathrm{x}<\mathrm{y}$ then not $(\mathrm{y}<\mathrm{x})$ ] must be valid. 
Not necessarily transitive: While WFR may be transitive, it is not necessary. Minimal Element: If $\mathrm{A}$ is any nonempty subset of a wellfounded set, there is an element in A such that $\mathrm{x}<\mathrm{y}$ for all $\mathrm{y}$ in $\mathrm{A}$. In other words, every nonempty subset of a wellfounded set contains a minimal element.

In computing logic time is quantized and is a well-founded set satisfying the properties - irreflexivity and asymmetry. Hence, we have a strong sense that time starts with a minimal element, the experienced time can be measured by units, and it has an arrow due to the asymmetry property. In Physics (both classical and quantum), time is not a well founded set. Time is a computable real number which is infinitely divisible [25,26]. Also calculus (continuity, differentiability and integrability ) of real numbers plays a fundamental role in the physics of dynamical systems. Time arrow has not yet been established and time is symmetric [26].

\subsection{Transfinite Induction Principle}

An arbitrary well- founded relation over a set of objects (nonnegarive integers, tuples, trees) gives rise to the "Transfinite Induction Principle" stated below: If for some property $\mathrm{P}$ under every $\mathrm{z} \varepsilon \mathrm{Z}, \mathrm{P}(\mathrm{z})$ (i.e. $\mathrm{z}$ has the property $\mathrm{P}$ ) $\mathrm{x}$ can be proved under the assumption that $\mathrm{p}\left(\mathrm{z}^{\prime}\right)$ holds for every $\mathrm{z}^{\prime}<\mathrm{z}$ then $\mathrm{P}(\mathrm{z})$ holds for all $\mathrm{z} \varepsilon \mathrm{Z}$. This principle plays a very crucial in the deductive foundations of computer programming to prove correctness and termination of programs not only over the integers, but also over other data structures -such as tuples and trees.

\subsection{Consequences of Infinite Divisibility of Time}

1. Since time is real and not a well-founded set, the transfinite induction principle (that permits inductive inference) fails. Therefore, quantum programs are not provable for correctness and termination using the classical generalised induction principle for nonnegative integers, tuples or trees.

2. Since time is not discrete, it has to be represented as computable real. That is there exists a computable sequence of rationals which converge effectively to a given value t. Thus we need to compute "bit by bit" the value of time that converges effectively within a radius of convergence.

3. Since time is infinitely divisible, the complexity of quantum computing becomes incomparable to the classical complexity results.

4. The recursive function over integers consists of the function algebra, consisting of some basic functions and closed under certain operations $[3,11,13,17]$ :

\{ZERO, SUCCESSOR, PREDECESSOR, PROJECTION, COMPOSITION, PRIMITIVE RECURSION, BOUNDED MINIMALIZATION\}. This can be generalised [3] to the primitive real class with:\{ZERO, SUCCESSOR, PREDECESSOR, PROJECTION, COMPOSITION, INTEGRATION, ZEROFINDING on REAL \} permitting us to define periodic functions like Sin, $\pi$, e. Also similar to the case of primitive recursive functions over integers that are not closed under minimalization, we also have the corresponding situation arising for integration. An important difference in real recursion is the replacement of primitive recursion by integration. Thus integrability becomes an important issue in dealing with complexity aspects over reals and complex numbers [40-43]. 
5. To reason about quantum programs, we need to develop the theory of recursive functions over the reals and an associated theory of programmability that can bridge with the theories of computational complexity, dynamical systems (classical and quantum), and numerical analysis.

6. Since time is real, we can convert an infinite time computation to a finite time by using Zeno squeezing oracle to be described in the next subsection. In fact time-line can be cut through to produce fractal time. This can result in statistically self-similar evolution $[5,25,27,28,29]$. In such a case, space and time should be appropriately defined for evaluating measures of complexity.

\subsection{Zeno Squeezing Oracle}

Turing defines an oracle [28] thus: An oracle is an agent or a "blackbox", which upon being consulted, supplies the true(correct) answers about mathematical or algorithmic or physical entities. Oracles though unrealizable in any universal computer, are useful to achieve the limit of an infinite computation. To conceive of such an oracle two time scales are introduced as below:

(i) an intrinsic time scale of the process of computation which tends to infinity and

(ii) an extrinsic time scale that remains finite for an external observer.

By using two such time scales, it is possible to produce within a finite proper time an output that would otherwise take an infinite time. This is called Zeno squeezing, since the infinite intrinsic time is squeezed to a finite extrinsic time limit. For example consider a time scale $t(i)$ belonging to the set of integers:

$\mathrm{t}(0)=0 ; \mathrm{t}(1)=1, \ldots, \mathrm{t}(\mathrm{k})=\mathrm{k}, \ldots \ldots$

Consider a scale $\mathrm{T}$ with $\mathrm{T}(0)=\mathrm{t}(0)=0 ; \mathrm{T}(\mathrm{i}>0)=\left[\mathrm{k}^{\mathrm{t}(\mathrm{i})}-1\right] /(\mathrm{k}-1)$ with $0<\mathrm{k}<1$. In the limit when $t$ (i) tends to $\infty$, the proper time $T(\infty)=1 /(1-k)$ remains finite.

A common feature of the Zeno oracle is that the valuation assigned to the intrinsic time $t$ decreases with its increasing value, reaching a finite extrinsic time limit when the intrinsic time is infinity. Thus time flow is non-uniform with a higher valuation for closer events and a lower valuation for events into a far future, much like the visual valuation of the size of objects as spatial distance varies from near to far. This is analogous to the non-Archimedian valuation used in p-adic system [7]. Perhaps, quantum systems may offer the possibility of devising oracles to squeeze time. This can result in statistically self- similar evolution locally at every point of time and scaling invariance analogous to Brownian motion and fractional Brownian motion.

\section{Concluding Remarks}

We explained some important reasons why quantum algorithms are difficult to design and implement. In particular, we explained the difficulty in seeking an appropriate structural transformation from a given problem domain to the non-dissipative quantum domain,failure of programmability and scalability. Since time in Physics is a real number quantum programs are not provable using transfinite induction principle. 


\section{References}

1. Ban, M: Information and entropy in Quantum measurement processes, International $\mathbf{J}$ Theoretical physics, 37 (1998) 2491-2537

2. Braun,D: Dissipative Quantum chaos and Decoherence,Springer, New York ( 2001)

3. Campagnolo, M.L.: The Complexity of real recursive functions, in Lecture Notes in Computer Science, Vol.2509: Springer Verlag, New York (2002) 1-14.

4. Deutsch,D.: Quantum Theory, the Church Turing principle and the universal quantum computer, Proc. Roy. soc. London, A 400 (1985) 97-117.

5. Feder,J: Fractals, Plenum Press, New York,(1988)

6. Feynman,R: Quantum Mechanical Computers, Foundations of Physics,16 (1986)507-531.

7. Gregory ,R.T and Krishnamurthy, E.V: Methods and Applications of Error-free Computation, Springer Verlag, New York ( 1984)

8. Gruska ,J: Quantum Computing, McGraw Hill, London (1999)

9. Hey,A.J.C., and. Allen, R.W: Feynman Lectures on Computation, Addison Wesley, New York, (1996)

10. Kitaev, A., Yu, Shen, A.H., and Vyalyi, M.N: Classical and Quantum Computation, American Mathematical Society, New York ( 2002)

11. Krishnamurthy, E.V.: Introductory Theory of Computer Science, Springer Verlag, New York, (1984)

12. Krishnamurthy, E.V.: Error-free Polynomial Matrix Computations, Springer Verlag, New York, (1985)

13. Krishnamurthy, E.V.: Parallel Processing, Addison Wesley, Reading, Mass( 1990)

14. Krishnamurthy E.V.: Complexity Issues in parallel and distributed Computing, Chapter 4, in Parallel and Distributed Computing Handbook, Ed. A. Zomaya, McGraw Hill, New York, (1996) 89-146

15. Krishnamurthy, E.V.: Computational power of quantum machines, quantum grammars, and feasible computation, International J. Modern Physics. C 9 (1998) 213-241.

16. Krishnamurthy, E.V: Integrability, Entropy and quantum computation, International J. Modern Physics. C10(1999) 1205-1228.

17. Krishnamurthy, E.V and Krishnamurthy, V:, Quantum Field theory and Computational Paradigms, International J Modern Physics. C12 (2001),1179-1205.

18. Krishnamurthy, E.V: Mapping, Programmability and Scalability of problems for quantum speed-up, International J. Modern Physics, in press.

19. Kroger, F: Temporal Logic of Programs, Springer Verlag, New York (1987)

20. Lamport, L: Time, Clocks, and the ordering of events in a distributed system, Comm. ACM,.21 (1978) 558-565.

21. Landauer, R: Is Quantum mechanics useful? Phil.Trans.Roy.Soc., Lond, A.353 (1995) 367-376

22. Manna, Z., and Waldinger,R.:The deductive foundations of Computer programming, Addison Wesley, Reading, (1993)

23. Nielsen, M.A., and Chuang, I.L.: Quantum Computation and Quantum Information, Cambridge University Press, Cambridge, (2000)

24. Niwa, J., Matsumoto. K., and Imai, H.: General purpose Parallel simulator for Quantum computing, Lecture Notes in Computer Science, Vol.2509,Springer Verlag, New York, (2002) 230-251.

25. Nottale, L.: Fractal Space-Time and Microphysics, World Scientific, Singapore (1993)

26. Park, D.: Time in Quantum mechanics, in Fundamental questions in quantum mechanics, Ed. L.M. Roth and A. Inomata, Gordon and Breach Science Publishers, London (1986)

27. Peitgen, H., Jurgens, H., and Saupe, D.: Fractals for the class room, Part 1, Springer Verlag, New York, (1991)

28. Svozil, K.: Randomness \& Undecidability in Physics,World Scientific, Singapore(1993)

29. Wolfram, S.: A New kind of Science, Wolfram Media Inc., Champaign, Ill,( 2002). 